OPEN ACCESS

Edited by:

Linda G.W. Kerkmeijer,

Radboud University Nijmegen Medical

Centre, Netherlands

Reviewed by:

X. Allen Li,

Medical College of Wisconsin,

United States

Dimitre Hristov

Stanford University, United States

*Correspondence:

Daniela Thorwarth

daniela.thorwarth@med.uni-

tuebingen.de

Specialty section:

This article was submitted to

Radiation Oncology,

a section of the journal

Frontiers in Oncology

Received: 27 November 2020 Accepted: 26 January 2021

Published: 08 March 2021

Citation:

Thorwarth D and Low DA (2021)

Technical Challenges of Real-Time Adaptive MR-Guided Radiotherapy.

Front. Oncol. 11:634507.

doi: 10.3389/fonc.2021.634507

\section{Technical Challenges of Real-Time Adaptive MR-Guided Radiotherapy}

\author{
Daniela Thorwarth ${ }^{1 *}$ and Daniel A. Low ${ }^{2}$ \\ 1 Section for Biomedical Physics, Department of Radiation Oncology, University of Tübingen, Tübingen, Germany, \\ ${ }^{2}$ Department of Radiation Oncology, University of California, Los Angeles, Los Angeles, CA, United States
}

In the past few years, radiotherapy (RT) has experienced a major technological innovation with the development of hybrid machines combining magnetic resonance (MR) imaging and linear accelerators. This new technology for MR-guided cancer treatment has the potential to revolutionize the field of adaptive RT due to the opportunity to provide highresolution, real-time MR imaging before and during treatment application. However, from a technical point of view, several challenges remain which need to be tackled to ensure safe and robust real-time adaptive MR-guided RT delivery. In this manuscript, several technical challenges to MR-guided RT are discussed. Starting with magnetic field strength tradeoffs, the potential and limitations for purely MR-based RT workflows are discussed. Furthermore, the current status of real-time 3D MR imaging and its potential for real-time $\mathrm{RT}$ are summarized. Finally, the potential of quantitative MR imaging for future biological RT adaptation is highlighted.

\section{Keywords: MR-linac, MR-guided radiotherapy, biologically adaptive radiotherapy, MR-only radiotherapy, online} adaptive radiotherapy, real-time adaptive radiotherapy

\section{INTRODUCTION}

The development of radiation therapy (RT) technology has enabled radiation oncologists to conform radiation doses to a level that was assumed to be physically impossible during the first 90 years of the field. Tumor margin prescriptions were developed to account for the differences between the radiation dose distribution and the patient's anatomy based on patient positioning errors, anatomical changes, and intrafraction motion (1). In-room imaging went a long way to reduce the margins needed to account for positioning and anatomical changes, but intrafraction motion remained a challenge due to the lack of real-time internal imaging of soft tissues $(2,3)$. This limitation was solved with the recent development of magnetic resonance (MR)-guided RT, defined as the integration of a radiation-delivery machine and an MR scanner $(4,5)$. While intra-fraction and real-time imaging became more straightforward, the improved soft tissue contrast of MR and the relatively low level of artifacts made this modality the first practical platform for adaptive RT $(6-12)$.

While MR imaging delivers no ionizing radiation, some acquisition protocols are limited due to tissue heating, which restricts some of the real-time imaging protocols, especially for high magnetic field systems. The clearance between the patient and the machine is also smaller than for conventional linear accelerators, limiting patient positioning strategies. The impact of the main 
magnetic field on the delivered radiation dose can be profound, and unlike computed tomography (CT), maintaining adequate spatial accuracy requires great care and needs to be checked routinely. Still, with all of these caveats, MR-guided RT (MRgRT) is likely to revolutionize some RT treatments, especially those that need high spatial resolution soft tissue imaging each fraction or real-time imaging for linear accelerator gating.

The aim of this review is to discuss technical challenges in the field of real-time adaptive MRgRT and to highlight current directions of research which reach out for new technical solutions to provide a basis for future clinical achievements in this field.

\section{MAGNETIC FIELD STRENGTH TRADEOFFS}

One of the more obvious differences between the commercial MRgRT systems is their main magnetic field strengths $(4,5)$. Current systems span the range of 0.35 to $1.5 \mathrm{~T}$, inviting the question of what, if any, are the tradeoffs between the different magnetic field strengths? Radiology's history of MR imaging main magnetic field strengths may imply that greater magnetic field strengths always provide better images than can be acquired at lower magnetic field strengths. Because the images are produced by the net polarization of water protons, which is proportional to the main magnetic field, the number of protons available to produce the radiofrequency $(\mathrm{RF})$ signal required for image acquisition increases with increasing field strength. All other things being equal, the subsequent signal to noise ratio (SNR) increases due to the increased number of polarized protons.

The purpose of MRgRT systems is to treat cancer, and since all commercial systems treat with $\mathrm{x}$-rays, generally accepted clinical quality and accuracy specifications, e.g., established by the International Commission on Radiation Units and Measurements (ICRU), should be met, as should imaging accuracy. The core functionality of MRgRT systems is not to mimic diagnostic systems, and as such the benchmark for their imaging performance should not come from diagnostic radiology requirements, but from radiation oncology requirements $(13,14)$.

The requirements of dose distribution accuracy and image fidelity can be examined independently. With respect to dose distribution accuracy, the AAPM stipulates that the overall accuracy goal is that the delivered dose should agree with the physical dose to within 5\%, a specification that includes uncertainties in machine calibration and dose calculation accuracy (15). While x-rays themselves are not impacted by the magnetic field, the secondary electrons are. When an external magnetic field is applied, the electrons are influenced by the Lorenz force, causing them to travel in a circle, but because of their many medium interactions, the overall paths are instead distorted in the direction of the Lorenz force. This distortion increases with increasing magnetic field. For larger radiation fields in a homogeneous water phantom, this distortion affects only the lateral beam penumbra. As the fields get small with respect to their secondary electron range, the entire high dose region distorts towards the Lorenz force direction $(16,17)$. When heterogeneities such as air cavities are encountered, the curved electron trajectories caused by the Lorentz force cause those secondary electrons to return to the exit cavity surface, substantially increasing the dose at those surfaces. These surfaces include tissue-air interfaces (such as bowel gas, the trachea, or nasal cavities) and tissue-lung interfaces, and the dose hot spots can be as large as $48 \%$ for a $1.5 \mathrm{~T}$ MRgRT system (18). State-of-the-art dose calculation software utilizes Monte Carlo transport calculations that model the influence of the magnetic fields, so the calculated dose can meet the accuracy requirements for a static patient, but changes in the heterogeneity distribution between the simulation scan and the patient's anatomy during the treatment can cause the doses at these interfaces to differ substantially from the calculated doses. That said, the relative dose heterogeneities can be somewhat compensated by overlapping beams from different directions. Finally, exit skin dose can exhibit the same behavior as internal heterogeneities, causing the skin exit dose to considerably higher than it would in a non-MRgRT treatment $(19,20)$.

Importantly, radiation dosimetry of air-filled ionization chambers is significantly influenced by the presence of a static magnetic field. To account for this effect during absolute dosimetry, dedicated field strength and chamber type and orientation specific correction factors need to be identified via measurements or simulations (21-25).

The imaging fidelity can be summarized as image quality for organ delineation, and geometric accuracy. It is generally considered that MRgRT systems provide image quality that is adequate for its intended purpose. MR images are generated using magnetic field gradients and an assumption of the knowledge of the relationship between the magnetic field strengths and position. Errors in this relationship cause the imaged features to appear offset from their actual positions. CT-based IGRT geometric alignment specification tolerance is $1 \mathrm{~mm}$ (26). Published spatial accuracy of the commercial MRgRT systems show that the $0.35 \mathrm{~T}$ system meets the $1 \mathrm{~mm}$ specification within $5 \mathrm{~cm}$ radius from isocenter and a $2 \mathrm{~mm}$ specification at $17.5 \mathrm{~cm}$ from isocenter (27), while the $1.5 \mathrm{~T}$ system has a $1.1 \mathrm{~mm}$ maximum spatial distortion within $20.0 \mathrm{~cm}$ from isocenter $(28,29)$.

Machine-based magnetic field errors are not the only source of MR image distortion. The patient chemical makeup will also modify the local magnetic field and therefore the apparent position of an imaged structure. Such susceptibility artifacts or chemical shifts lead to shifts in the imaged positions of anatomical structures which are proportional to the magnetic field. For human tissues, these can be in the order of millimeters for $1.5 \mathrm{~T}$ scanners (30), while the same artifact at $0.35 \mathrm{~T}$ would be much lower. For specific sequences, the susceptibility artifacts can be reduced by increasing the RF bandwidth, which has the corresponding side-effect of reducing SNR, reducing, but not eliminating the advantage of the increased field strength.

Finally, the radiofrequency energy emitted by the MR scanner is absorbed by the human body, heating the body. 
The term used to describe this for clinical MR scans is the specific absorption rate (SAR). The amount of heating is proportional to the square of the magnetic field strength (31), so limiting the SAR will be more challenging for the higher field MRgRT systems. Limiting the SAR may be most challenging when conducting real-time imaging for purposes of linear accelerator gating. Two of the "selling points" of MR are that it does not deliver ionizing radiation and that it can provide real-time internal imaging, so restricting this imaging due to SAR concerns would reduce the perceived benefit of MRgRT.

\section{REAL-TIME MR AUTO-SEGMENTATION}

Current clinical experience of online adaptive MRgRT shows that one of the main bottle necks is the lack of fast and accurate segmentation of MR images. As the requirement for real-time adaptive MRgRT is to robustly provide MR-based structure delineations in the order of seconds, deep learning (DL) approaches have been investigated recently by several groups (32-36). Most DL models for auto-contouring were trained so far for the pelvic region providing to generate organ structures based on MR images $(32,34)$. Additionally, DL concepts for contour propagation from simulation images to daily MR have been proposed recently (37). Since adaptive MRgRT is a novel clinical application, annotated MR data for model training and validation is sparse, thus alternative approaches such as crossmodality learning have been explored (38). Even though numerous challenges remain concerning real-time MR-based auto-segmentation, first investigations regarding the clinical implementation have reported fast (few seconds) and robust use in MRgRT of prostate cancer (39).

\section{MR-ONLY PLANNING}

During real-time adaptive MRgRT, treatment planning as well as dose calculation need to be conducted for every RT fraction, but a CT simulation is no longer available. Consequently, approaches for MR-based dose planning-so called MR-only planning workflows - have been proposed to support real-time adaptive MRgRT.

MR-only planning in combination with MR-simulation for RT planning without the need of additional planning CT has been previously proposed $(40,41)$. Early on, mechanistic models using dedicated MR sequences, such as e.g., Dixon-based sequences, were proposed to generate synthetic CT data sets based on MR imaging data (42). Alternative approaches proposed voxel-intensity based approaches to translate MR signal values into synthetic CT readings (42). Dosimetric studies analyzing the accuracy of radiation dose calculation using synthetic CT reported dose differences on the order of $0.5 \%$ relative to CT-based dose simulation (43). Today, several commercial products for synthetic CT generation are available and studies reporting first clinical experience using MR-only simulation for RT planning have been recently published (44).
Because the acquisition of dedicated MR sequences for synthetic CT determination is time consuming, online adaptive MRgRT in today's clinical practice mostly relies on extremely simplified methods to generate synthetic CT information, such as bulk density assignments to anatomical structures. This simplification may compromise the dosimetric accuracy as currently robust conversion approaches from MR to CT are lacking. To overcome this, several groups have recently proposed deep learning models for the calculation of synthetic CT data sets based on anatomical MR imaging which has shown to be a timeefficient and robust approach (45-48). Dosimetric evaluations have shown promising results in terms of dose differences of 0 $0.5 \%(49,50)$. However, online quality assurance of synthetic CT seems to be challenging and bears dosimetric risks especially when it comes to the anatomical location and electron density assignment of bony structures. The use of undistorted MR images for synthetic CT generation is crucial in the light of real-time high precision MR-guidance. Nevertheless, the use of artificial intelligence (AI) tools for MR-only workflows may open new opportunities for real-time adaptive MRgRT using hybrid MR-linacs.

\section{REAL-TIME MRI}

One of the most compelling features of MRgRT is the ability to conduct real-time imaging $(51,52)$. Real-time imaging provides unparalleled visualization of internal organs to enable the clinicians to monitor and ultimately limit the impact of intra-fraction motion. This motion may be due to peristalsis, bladder filling, or breathing. The MRgRT system will provide a sequence of images, typically at a few Hertz. This sequence is typically started immediately after any setup images are acquired where the tumor is identified, and the patient moved to account for relative shifts or deformations. If the motion is due to breathing, the sequence is typically visualized for a few breathing cycles to determine the amount of motion. If gating is available and desired, a gating window is defined by segmenting the target or a suitable surrogate and applying a margin to act as a gating structure. The MRgRT system then tracks that gating structure for each image frame and monitors whether the structure is within the gating window, typically to within a preselected percentage. Note that this process is currently $2 \mathrm{D}$, due to a lack of commercially available real-time $3 \mathrm{D}$ imaging sequences. Recent studies however showed promising approaches towards realtime 3D MR image acquisition (53-56) and reconstruction (57).

A critical concern is the latency between the time, the images are acquired and the time the machine or operator can respond to an undesired motion. This is related to the amount of time required to acquire the image data, to reconstruct the data, and to analyze the data and determine that a significant deviation has occurred. The latter could be the time required for the system to conduct the contouring, the determination that the motion should trigger a change in machine state and implement that change (beam on or off) or the time a human operator would take to evaluate and manually change the machine state. The latency of the image acquisition step is typically assumed to be 
approximately half of the image acquisition time because the image is expected to reflect the average state during the acquisition time. The remaining latency sources are functions of the hardware and software that the manufacturer employs. These times should be short, especially for free breathing motion gating, where multiple gating events will take place during a treatment.

Recent studies have shown latency times of real-time 3D MR imaging on MR-linac systems of 300-500 ms (58). Quality assurance to verify real-time interventions should be performed using a dynamic phantom that contains MR-imageable structures and the ability to do both point (ion chamber) and area (film) dosimetry. An end-to-end test of a gated treatment that uses clinically realistic treatment times and gating windows will determine if the latency significantly degrades the treatment dose accuracy (59).

\section{ONLINE ADAPTIVE RT}

With the advent of 4D-MR imaging with minimal latency times, real-time adaptive RT seems to be one of the next technological steps of MR-guided radiotherapy. Consequently, it might be possible to irradiate moving targets with highest precision using MLC-tracking based on real-time MR readings. MLC-tracking based on CBCT imaging has been proposed earlier and proven to be suitable for clinical usage (60). A major challenge of real-time adaptive RT is the methodology of real-time dose calculation or reconstruction. Fast et al. (61) proposed a tool for online dose reconstruction which determined the delivered dose based on pre-calculated dose influence data in less than $10 \mathrm{~ms}$. After initial investigations of online dose reconstruction based on 2D cine MR images (62) and $3 \mathrm{D}$ cine $\mathrm{MR}$ in addition to treatment log files (63), recent studies proposed deep learning strategies to empower real-time dose calculation and motion prediction $(64,65)$. Even though proposed for offline planning, methods for deep learning-based dose prediction seem to be promising tools to support real-time dose reconstruction $(66,67)$. In the light of current trends for reduced number of $\mathrm{RT}$ fractions, dose adaptation and calculation based on real-time anatomical information gets more and more important.

Accurate dose assessment of fractionated RT requires deformable dose accumulation for targets and OARs. So far, no clinically usable solution has been proposed for this problem. Therefore, robust algorithms for $4 \mathrm{D}$ dose accumulation are required to provide precise voxel-readings of recorded, locally varying dose distributions for better TCP and NTCP estimation (68).

Nevertheless, clinical real-time adaptive MRgRT needs thorough quality assurance and testing. To date, dedicated end-to-end tests were proposed to specifically test certain aspects of adaptive MR-guided RT (7, 59, 69-71). Future endto-end test developments may focus on ways to evaluate realtime imaging, dose calculation, and accumulation. Furthermore, mechanisms that ensure robust and safe radiation delivery need to be implemented in real-time MR guided workflows.

\section{FUNCTIONAL IMAGING}

In addition to the enormous potential of MRgRT for geometrical precision and adaptation in real-time, functional MR data has been shown by several studies to be prognostic for outcome after RT in different tumor entities $(72,73)$. Consequently, interventions steered by functional MR imaging biomarkers seem to be one of the most promising concepts towards personalized, biologically individualized RT. Even though prognostic information using functional MR imaging may also be gathered with state-of-the-art diagnostic MR systems which do not suffer from hardware limitations such as the hybrid MR-linac scanners (74), biological real-time adaptation such as image biomarker guided dose painting to overcome local tumor radiation resistances can only be realized on hybrid MR-linacs (75). Biological RT individualization in terms of dose adaptation based on imaging information requires the measurement of quantitative imaging biomarkers (76). Recent studies have shown that quantitative MR imaging is possible using hybrid MR-linacs (77). Diffusion-weighted imaging (DWI), for example, can be implemented such that robust quantitative diffusion data can be measured with high repeatability and reproducibility (77). A major challenge for using quantitative biomarkers in observational and also interventional multi-center MR-linac studies will be the validation of imaging protocols for reproducibility of quantitative imaging in order to prove that quantitative imaging biomarkers are comparable between centers (78). Furthermore, test-retest studies to assess the level of repeatability will be prerequisites for future quantitative imaging studies in different tumor entities. So far, most studies have focused on quantitative imaging assessments and on investigating prognostic value of DWI $(72,74,77,79-81)$. A further challenge will be the realization of functional interventions. Currently, echo planar MR imaging (EPI) techniques are mostly used for DWI even though these are known to be susceptible for geometrical distortions (82). However, dose painting based on functional MR data requires geometrical accuracy. Consequently, current research strategies in this field include investigation of alternative MR imaging techniques, e.g., turbo spin echo (TSE) based sequences such as SPLICE (83) or strategies to correct for geometrical distortions (82). Nevertheless, hybrid MR-linacs are a major technological innovation towards real-time biological adaptation of RT aiming for increasing tumor control rates in different cancer types in the future.

\section{DISCUSSION}

MR-guided RT offers high-resolution real time MR imaging before and during RT and allows thus to adapt for inter- and intra-fraction changes. Consequently, smaller target margins and potentially better organ-at-risk sparing may be possible with MRgRT, opening new horizons towards single or few fraction RT delivery (84). For real-time MR-guidance, many involved steps require automatization. Researchers in different sub-fields have started to automatize and speed-up processes using AI methods. However, to generate robust and intelligent models which can assist with 
treatment decisions, large sets of curated, standardized and well documented data are needed for model training and validation.

Furthermore, there is evidence, that biological characteristics of the tumor microenvironment play an important role in terms of radiation resistance. Consequently, quantitative MR imaging biomarkers need to be identified as predictive for RT outcome, validated in phantom and clinical studies and might then in the future qualify for interventional, quantitative MR based RT studies.

Ultimately, all technical solutions developed to overcome challenges related to real-time adaptive MR-guided RT deserve intensive clinical validation before unsupervised usage in routine MRgRT.

\section{REFERENCES}

1. van Herk M. Errors and margins in radiotherapy. Semin Radiat Oncol (2004) 14(1):52-64. doi: 10.1053/j.semradonc.2003.10.003

2. Gregoire V, Guckenberger M, Haustermans K, Lagendijk JJW, Menard C, Potter R, et al. Image guidance in radiation therapy for better cure of cancer. Mol Oncol (2020) 14(7):1470-91. doi: 10.1002/1878-0261.12751

3. Hall WA, Paulson ES, van der Heide UA, Fuller CD, Raaymakers BW, Lagendijk JJW, et al. The transformation of radiation oncology using realtime magnetic resonance guidance: A review. Eur J Cancer (2019) 122:42-52. doi: 10.1016/j.ejca.2019.07.021

4. Winkel D, Bol GH, Kroon PS, van Asselen B, Hackett SS, WerensteijnHoningh AM, et al. Adaptive radiotherapy: The Elekta Unity MR-linac concept. Clin Transl Radiat Oncol (2019) 18:54-9. doi: 10.1016/ j.ctro.2019.04.001

5. Kluter S. Technical design and concept of a $0.35 \mathrm{~T}$ MR-Linac. Clin Transl Radiat Oncol (2019) 18:98-101. doi: 10.1016/j.ctro.2019.04.007

6. Chen AM, Hsu S, Lamb J, Yang Y, Agazaryan N, Steinberg ML, et al. MRIguided radiotherapy for head and neck cancer: initial clinical experience. Clin Transl Oncol (2018) 20(2):160-8. doi: 10.1007/s12094-017-1704-4

7. Lamb JM, Ginn JS, O'Connell DP, Agazaryan N, Cao M, Thomas DH, et al. Dosimetric validation of a magnetic resonance image gated radiotherapy system using a motion phantom and radiochromic film. J Appl Clin Med Phys (2017) 18(3):163-9. doi: 10.1002/acm2.12088

8. Tyran M, Jiang N, Cao M, Raldow A, Lamb JM, Low D, et al. Retrospective evaluation of decision-making for pancreatic stereotactic MR-guided adaptive radiotherapy. Radiother Oncol (2018) 129(2):319-25. doi: 10.1016/ j.radonc.2018.08.009

9. Bertelsen AS, Schytte T, Moller PK, Mahmood F, Riis HL, Gottlieb KL, et al. First clinical experiences with a high field 1.5 T MR linac. Acta Oncol (2019) 58(10):1352-7. doi: 10.1080/0284186X.2019.1627417

10. Werensteijn-Honingh AM, Kroon PS, Winkel D, Aalbers EM, van Asselen B, Bol GH, et al. Feasibility of stereotactic radiotherapy using a 1.5T MR-linac: Multi-fraction treatment of pelvic lymph node oligometastases. Radiother Oncol (2019) 134:50-4. doi: 10.1016/j.radonc.2019.01.024

11. Winkel D, Bol GH, Werensteijn-Honingh AM, Intven MPW, Eppinga WSC, Hes J, et al. Target coverage and dose criteria based evaluation of the first clinical 1.5T MR-linac SBRT treatments of lymph node oligometastases compared with conventional CBCT-linac treatment. Radiother Oncol (2020) 146:118-25. doi: 10.1016/j.radonc.2020.02.011

12. Kluter S, Katayama S, Spindeldreier CK, Koerber SA, Major G, Alber M, et al. First prospective clinical evaluation of feasibility and patient acceptance of magnetic resonance-guided radiotherapy in Germany. Strahlenther Onkol (2020) 196(8):691-8. doi: 10.1007/s00066-020-01578-z

13. Paulson ES, Crijns SP, Keller BM, Wang J, Schmidt MA, Coutts G, et al. Consensus opinion on MRI simulation for external beam radiation treatment planning. Radiother Oncol (2016) 121(2):187-92. doi: 10.1016/j.radonc.2016.09.018

14. Menard C, van der Heide UA. Introduction: Magnetic resonance imaging comes of age in radiation oncology. Semin Radiat Oncol (2014) 24(3):149-50. doi: 10.1016/j.semradonc.2014.02.001

\section{AUTHOR CONTRIBUTIONS}

DT and DL equally contributed to writing and proof-reading of this manuscript. All authors contributed to the article and approved the submitted version.

\section{FUNDING}

The Tübingen MR-Linac program received funding from the German Research Council (DFG, grants no. ZI 736/2-1, TH 1528/6-1). We acknowledge support by Open Access Publishing Fund of University of Tübingen.

15. Klein EE, Hanley J, Bayouth J, Yin FF, Simon W, Dresser S, et al. Task Group 142 report: quality assurance of medical accelerators. Med Phys (2009) 36 (9):4197-212. doi: 10.1118/1.3190392

16. Raaymakers BW, Raaijmakers AJ, Kotte AN, Jette D, Lagendijk JJ. Integrating a MRI scanner with a $6 \mathrm{MV}$ radiotherapy accelerator: dose deposition in a transverse magnetic field. Phys Med Biol (2004) 49(17):4109-18. doi: 10.1088/ 0031-9155/49/17/019

17. Friedel M, Nachbar M, Monnich D, Dohm O, Thorwarth D. Development and validation of a 1.5 T MR-Linac full accelerator head and cryostat model for Monte Carlo dose simulations. Med Phys (2019) 46(11):5304-13. doi: $10.1002 / \mathrm{mp} .13829$

18. Raaijmakers AJ, Raaymakers BW, Lagendijk JJ. Integrating a MRI scanner with a $6 \mathrm{MV}$ radiotherapy accelerator: dose increase at tissue-air interfaces in a lateral magnetic field due to returning electrons. Phys Med Biol (2005) 50 (7):1363-76. doi: 10.1088/0031-9155/50/7/002

19. Nachbar M, Monnich D, Boeke S, Gani C, Weidner N, Heinrich V, et al Partial breast irradiation with the 1.5 T MR-Linac: First patient treatment and analysis of electron return and stream effects. Radiother Oncol (2020) 145:305. doi: 10.1016/j.radonc.2019.11.025

20. Nachbar M, Monnich D, Kalwa P, Zips D, Thorwarth D, Gani C. Comparison of treatment plans for a high-field MRI-linac and a conventional linac for esophageal cancer. Strahlenther Onkol (2019) 195(4):327-34. doi: 10.1007/ s00066-018-1386-z

21. Pojtinger S, Dohm OS, Kapsch RP, Thorwarth D. Ionization chamber correction factors for MR-linacs. Phys Med Biol (2018) 63(11):11NT03. doi: 10.1088/1361-6560/aac4f2

22. Pojtinger S, Kapsch RP, Dohm OS, Thorwarth D. A finite element method for the determination of the relative response of ionization chambers in MRlinacs: simulation and experimental validation up to 1.5 T. Phys Med Biol (2019) 64(13):135011. doi: 10.1088/1361-6560/ab2837

23. Pojtinger S, Nachbar M, Ghandour S, Pisaturo O, Pachoud M, Kapsch RP, et al. Experimental determination of magnetic field correction factors for ionization chambers in parallel and perpendicular orientations. Phys Med Biol (2020) 65(24):245044. doi: 10.1088/1361-6560/abca06

24. O'Brien DJ, Roberts DA, Ibbott GS, Sawakuchi GO. Reference dosimetry in magnetic fields: formalism and ionization chamber correction factors. Med Phys (2016) 43(8):4915. doi: 10.1118/1.4959785

25. Meijsing I, Raaymakers BW, Raaijmakers AJ, Kok JG, Hogeweg L, Liu B, et al. Dosimetry for the MRI accelerator: the impact of a magnetic field on the response of a Farmer NE2571 ionization chamber. Phys Med Biol (2009) 54 (10):2993-3002. doi: 10.1088/0031-9155/54/10/002

26. Bissonnette JP, Balter PA, Dong L, Langen KM, Lovelock DM, Miften M, et al. Quality assurance for image-guided radiation therapy utilizing CT-based technologies: a report of the AAPM TG-179. Med Phys (2012) 39(4):194663. doi: 10.1118/1.3690466

27. Ginn JS, Agazaryan N, Cao M, Baharom U, Low DA, Yang Y, et al. Characterization of spatial distortion in a $0.35 \mathrm{~T}$ MRI-guided radiotherapy system. Phys Med Biol (2017) 62(11):4525-40. doi: 10.1088/1361-6560/aa6ela

28. Snyder JE, St-Aubin J, Yaddanapudi S, Boczkowski A, Dunkerley DAP, Graves SA, et al. Commissioning of a 1.5T Elekta Unity MR-linac: A single institution 
experience. J Appl Clin Med Phys (2020) 21(7):160-72. doi: 10.1002/ acm2.12902

29. Tijssen RHN, Philippens MEP, Paulson ES, Glitzner M, Chugh B, Wetscherek A, et al. MRI commissioning of 1.5T MR-linac systems - a multi-institutional study. Radiother Oncol (2019) 132:114-20. doi: 10.1016/j.radonc.2018.12.011

30. Hasler SW, Bernchou U, Bertelsen A, van Veldhuizen E, Schytte T, Hansen $\mathrm{VN}$, et al. Tumor-site specific geometric distortions in high field integrated magnetic resonance linear accelerator radiotherapy. Phys Imaging Radiat Oncol (2020) 15:100-4. doi: 10.1016/j.phro.2020.07.007

31. Bottomley PA. Turning up the heat on MRI. J Am Coll Radiol (2008) 5 (7):853-5. doi: 10.1016/j.jacr.2008.04.003

32. Elguindi S, Zelefsky MJ, Jiang J, Veeraraghavan H, Deasy JO, Hunt MA, et al. Deep learning-based auto-segmentation of targets and organs-at-risk for magnetic resonance imaging only planning of prostate radiotherapy. Phys Imaging Radiat Oncol (2019) 12:80-6. doi: 10.1016/j.phro.2019.11.006

33. Morris ED, Ghanem AII, Dong M, Pantelic MV, Walker E4M, Glide-Hurst CK. Cardiac substructure segmentation with deep learning for improved cardiac sparing. Med Phys (2020) 47(2):576-86. doi: 10.1002/mp.13940

34. Wang J, Lu J, Qin G, Shen L, Sun Y, Ying H, et al. Technical Note: A deep learning-based autosegmentation of rectal tumors in MR images. Med Phys (2018) 45(6):2560-4. doi: 10.1002/mp.12918

35. Chen Y, Ruan D, Xiao J, Wang L, Sun B, Saouaf R, et al. Fully automated multiorgan segmentation in abdominal magnetic resonance imaging with deep neural networks. Med Phys (2020) 47(10):4971-82. doi: 10.1002/ mp. 14429

36. Fu Y, Mazur TR, Wu X, Liu S, Chang X, Lu Y, et al. A novel MRI segmentation method using CNN-based correction network for MRI-guided adaptive radiotherapy. Med Phys (2018) 45(11):5129-37. doi: 10.1002/mp.13221

37. Eppenhof KAJ, Maspero M, Savenije MHF, de Boer JCJ, van der Voort van Zyp JRN, Raaymakers BW, et al. Fast contour propagation for MR-guided prostate radiotherapy using convolutional neural networks. Med Phys (2020) 47(3):1238-48. doi: 10.1002/mp.13994

38. Kieselmann JP, Fuller CD, Gurney-Champion OJ, Oelfke U. Cross-modality deep learning: Contouring of MRI data from annotated CT data only. Med Phys (2020). doi: 10.1002/mp.14619

39. Savenije MHF, Maspero M, Sikkes GG, van der Voort van Zyp JRN, AN TJK, Bol GH, et al. Clinical implementation of MRI-based organs-at-risk autosegmentation with convolutional networks for prostate radiotherapy. Radiat Oncol (2020) 15(1):104. doi: 10.1186/s13014-020-01528-0

40. Glide-Hurst CK, Low DA, Orton CG. Point/Counterpoint. MRI/CT is the future of radiotherapy treatment planning. Med Phys (2014) 41(11):110601. doi: 10.1118/1.4894495

41. Owrangi AM, Greer PB, Glide-Hurst CK. MRI-only treatment planning: benefits and challenges. Phys Med Biol (2018) 63(5):05TR01. doi: 10.1088/ 1361-6560/aaaca4

42. Edmund JM, Nyholm T. A review of substitute CT generation for MRI-only radiation therapy. Radiat Oncol (2017) 12(1):28. doi: 10.1186/s13014-016$0747-\mathrm{y}$

43. Tyagi N, Fontenla S, Zhang J, Cloutier M, Kadbi M, Mechalakos J, et al. Dosimetric and workflow evaluation of first commercial synthetic CT software for clinical use in pelvis. Phys Med Biol (2017) 62(8):2961-75. doi: 10.1088/ 1361-6560/aa5452

44. Tyagi N, Zelefsky MJ, Wibmer A, Zakian K, Burleson S, Happersett L, et al. Clinical experience and workflow challenges with magnetic resonance-only radiation therapy simulation and planning for prostate cancer. Phys Imaging Radiat Oncol (2020) 16:43-9. doi: 10.1016/j.phro.2020.09.009

45. Maspero M, Bentvelzen LG, Savenije MHF, Guerreiro F, Seravalli E, Janssens GO, et al. Deep learning-based synthetic CT generation for paediatric brain MR-only photon and proton radiotherapy. Radiother Oncol (2020) 153:197204. doi: $10.1016 /$ j.radonc.2020.09.029

46. Klages P, Benslimane I, Riyahi S, Jiang J, Hunt M, Deasy JO, et al. Patch-based generative adversarial neural network models for head and neck MR-only planning. Med Phys (2020) 47(2):626-42. doi: 10.1002/mp.13927

47. Olin AB, Hansen AE, Rasmussen JH, Ladefoged CN, Berthelsen AK, Hakansson K, et al. Feasibility of Multiparametric Positron Emission Tomography/Magnetic Resonance Imaging as a One-Stop Shop for Radiation Therapy Planning for Patients with Head and Neck Cancer. Int $J$
Radiat Oncol Biol Phys (2020) 108(5):1329-38. doi: 10.1016/j.ijrobp. 2020.07.024

48. Cusumano D, Lenkowicz J, Votta C, Boldrini L, Placidi L, Catucci F, et al. A deep learning approach to generate synthetic CT in low field MR-guided adaptive radiotherapy for abdominal and pelvic cases. Radiother Oncol (2020). doi: 10.1016/j.radonc.2020.10.018

49. Dinkla AM, Florkow MC, Maspero M, Savenije MHF, Zijlstra F, Doornaert $\mathrm{PAH}$, et al. Dosimetric evaluation of synthetic CT for head and neck radiotherapy generated by a patch-based three-dimensional convolutional neural network. Med Phys (2019) 46(9):4095-104. doi: 10.1002/mp.13663

50. Dinkla AM, Wolterink JM, Maspero M, Savenije MHF, Verhoeff JJC, Seravalli E, et al. MR-Only Brain Radiation Therapy: Dosimetric Evaluation of Synthetic CTs Generated by a Dilated Convolutional Neural Network. Int J Radiat Oncol Biol Phys (2018) 102(4):801-12. doi: 10.1016/j.jirobp.2018.05.058

51. Glitzner M, Crijns SP, de Senneville BD, Kontaxis C, Prins FM, Lagendijk JJ, et al. On-line MR imaging for dose validation of abdominal radiotherapy. Phys Med Biol (2015) 60(22):8869-83. doi: 10.1088/0031-9155/60/22/8869

52. Stemkens B, Paulson ES, Tijssen RHN. Nuts and bolts of 4D-MRI for radiotherapy. Phys Med Biol (2018) 63(21):21TR01. doi: 10.1088/1361$6560 /$ aae56d

53. Ginn JS, Low DA, Lamb JM, Ruan D. A motion prediction confidence estimation framework for prediction-based radiotherapy gating. Med Phys (2020) 47(8):3297-304. doi: 10.1002/mp.14236

54. Ginn JS, Ruan D, Low DA, Lamb JM. An image regression motion prediction technique for MRI-guided radiotherapy evaluated in single-plane cine imaging. Med Phys (2020) 47(2):404-13. doi: 10.1002/mp.13948

55. Ginn JS, Ruan D, Low DA, Lamb JM. Multislice motion modeling for MRIguided radiotherapy gating. Med Phys (2019) 46(2):465-74. doi: 10.1002/ mp. 13350

56. Han F, Zhou Z, Du D, Gao Y, Rashid S, Cao M, et al. Respiratory motionresolved, self-gated 4D-MRI using Rotating Cartesian K-space (ROCK): Initial clinical experience on an MRI-guided radiotherapy system. Radiother Oncol (2018) 127(3):467-73. doi: 10.1016/j.radonc.2018.04.029

57. Terpstra ML, Maspero M, d'Agata F, Stemkens B, Intven MPW, Lagendijk JJW, et al. Deep learning-based image reconstruction and motion estimation from undersampled radial k-space for real-time MRI-guided radiotherapy. Phys Med Biol (2020) 65(15):155015. doi: 10.1088/1361-6560/ab9358

58. Borman PTS, Tijssen RHN, Bos C, Moonen CTW, Raaymakers BW, Glitzner M. Characterization of imaging latency for real-time MRI-guided radiotherapy. Phys Med Biol (2018) 63(15):155023. doi: 10.1088/1361-6560/aad2b7

59. Stark LS, Andratschke N, Baumgartl M, Bogowicz M, Chamberlain M, Dal Bello R, et al. Dosimetric and geometric end-to-end accuracy of a magnetic resonance guided linear accelerator. Phys Imaging Radiat Oncol (2020) 16:109-12. doi: 10.1016/j.phro.2020.09.013

60. Keall PJ, Colvill E, O'Brien R, Ng JA, Poulsen PR, Eade T, et al. The first clinical implementation of electromagnetic transponder-guided MLC tracking. Med Phys (2014) 41(2):020702. doi: 10.1118/1.4862509

61. Fast MF, Kamerling CP, Ziegenhein P, Menten MJ, Bedford JL, Nill S, et al. Assessment of MLC tracking performance during hypofractionated prostate radiotherapy using real-time dose reconstruction. Phys Med Biol (2016) 61 (4):1546-62. doi: 10.1088/0031-9155/61/4/1546

62. Kontaxis C, Bol GH, Stemkens B, Glitzner M, Prins FM, Kerkmeijer LGW, et al. Towards fast online intrafraction replanning for free-breathing stereotactic body radiation therapy with the MR-linac. Phys Med Biol (2017) 62(18):7233-48. doi: 10.1088/1361-6560/aa82ae

63. Kontaxis C, de Muinck Keizer DM, Kerkmeijer LGW, Willigenburg T, den Hartogh MD, van der Voort van Zyp JRN, et al. Delivered dose quantification in prostate radiotherapy using online $3 \mathrm{D}$ cine imaging and treatment log files on a combined $1.5 \mathrm{~T}$ magnetic resonance imaging and linear accelerator system. Phys Imaging Radiat Oncol (2020) 15:23-9. doi: 10.1016/ j.phro.2020.06.005

64. Kontaxis C, Bol GH, Lagendijk JJW, Raaymakers BW. DeepDose: Towards a fast dose calculation engine for radiation therapy using deep learning. Phys Med Biol (2020) 65(7):075013. doi: 10.1088/1361-6560/ab7630

65. Thomas MA, Olick-Gibson J, Fu Y, Parikh PJ, Green O, Yang D. Using prediction models to evaluate magnetic resonance image guided radiation therapy plans. Phys Imaging Radiat Oncol (2020) 16:99-102. doi: 10.1016/ j.phro.2020.10.002 
66. Nguyen D, Long T, Jia X, Lu W, Gu X, Iqbal Z, et al. A feasibility study for predicting optimal radiation therapy dose distributions of prostate cancer patients from patient anatomy using deep learning. Sci Rep (2019) 9(1):1076. doi: 10.1038/s41598-018-37741-X

67. Chen X, Men K, Li Y, Yi J, Dai J. A feasibility study on an automated method to generate patient-specific dose distributions for radiotherapy using deep learning. Med Phys (2019) 46(1):56-64. doi: 10.1002/mp.13262

68. Valdes G, Lee C, Tenn S, Lee P, Robinson C, Iwamoto K, et al. The relative accuracy of $4 \mathrm{D}$ dose accumulation for lung radiotherapy using rigid dose projection versus dose recalculation on every breathing phase. Med Phys (2017) 44(3):1120-7. doi: 10.1002/mp.12069

69. Monnich D, Winter J, Nachbar M, Kunzel L, Boeke S, Gani C, et al. Quality assurance of IMRT treatment plans for a 1.5 T MR-linac using a 2D ionization chamber array and a static solid phantom. Phys Med Biol (2020) 65 (16):16NT01. doi: 10.1088/1361-6560/aba5ec

70. Elter A, Dorsch S, Mann P, Runz A, Johnen W, Spindeldreier CK, et al. Endto-end test of an online adaptive treatment procedure in MR-guided radiotherapy using a phantom with anthropomorphic structures. Phys Med Biol (2019) 64(22):225003. doi: 10.1088/1361-6560/ab4d8e

71. Cunningham JM, Barberi EA, Miller J, Kim JP, Glide-Hurst CK. Development and evaluation of a novel MR-compatible pelvic end-to-end phantom. J Appl Clin Med Phys (2019) 20(1):265-75. doi: 10.1002/acm2.12455

72. Leibfarth S, Winter RM, Lyng H, Zips D, Thorwarth D. Potentials and challenges of diffusion-weighted magnetic resonance imaging in radiotherapy. Clin Transl Radiat Oncol (2018) 13:29-37. doi: 10.1016/j.ctro.2018.09.002

73. Mahmood F, Johannesen HH, Geertsen P, Hansen RH. Repeated diffusion MRI reveals earliest time point for stratification of radiotherapy response in brain metastases. Phys Med Biol (2017) 62(8):2990-3002. doi: 10.1088/1361$6560 / \mathrm{aa} 5249$

74. Kooreman ES, van Houdt PJ, Keesman R, Pos FJ, van Pelt VWJ, Nowee ME, et al. ADC measurements on the Unity MR-linac - A recommendation on behalf of the Elekta Unity MR-linac consortium. Radiother Oncol (2020) 153:106-13. doi: 10.1016/j.radonc.2020.09.046

75. Thorwarth D, Ege M, Nachbar M, Mönnich D, Gani C, Zips D, et al. Quantitative magnetic resonance imaging on hybrid magnetic resonance linear accelerators: Perspective on technical and clinical validation. Phys Imaging Radiat Oncol (2020) 16:69-73. doi: 10.1016/j.phro.2020.09.007

76. Gurney-Champion OJ, Mahmood F, van Schie M, Julian R, George B, Philippens MEP, et al. Quantitative imaging for radiotherapy purposes. Radiother Oncol (2020) 146:66-75. doi: 10.1016/j.radonc.2020.01.026

77. Kooreman ES, van Houdt PJ, Nowee ME, van Pelt VWJ, Tijssen RHN, Paulson ES, et al. Feasibility and accuracy of quantitative imaging on a $1.5 \mathrm{~T}$
MR-linear accelerator. Radiother Oncol (2019) 133:156-62. doi: 10.1016/ j.radonc.2019.01.011

78. van Houdt PJ, Kallehauge JF, Tanderup K, Nout R, Zaletelj M, Tadic T, et al. Phantom-based quality assurance for multicenter quantitative MRI in locally advanced cervical cancer. Radiother Oncol (2020) 153:114-21. doi: 10.1016/ j.radonc.2020.09.013

79. Gao Y, Han F, Zhou Z, Cao M, Kaprealian T, Kamrava M, et al. Distortionfree diffusion MRI using an MRI-guided Tri-Cobalt 60 radiotherapy system: Sequence verification and preliminary clinical experience. Med Phys (2017) 44 (10):5357-66. doi: 10.1002/mp.12465

80. Yang Y, Cao M, Sheng K, Gao Y, Chen A, Kamrava M, et al. Longitudinal diffusion MRI for treatment response assessment: Preliminary experience using an MRI-guided tri-cobalt 60 radiotherapy system. Med Phys (2016) 43 (3):1369-73. doi: 10.1118/1.4942381

81. Shaverdian N, Yang Y, Hu P, Hart S, Sheng K, Lamb J, et al. Feasibility evaluation of diffusion-weighted imaging using an integrated MRIradiotherapy system for response assessment to neoadjuvant therapy in rectal cancer. Br J Radiol (2017) 90(1071):20160739. doi: 10.1259/ bjr.20160739

82. Winter RM, Schmidt H, Leibfarth S, Zwirner K, Welz S, Schwenzer NF, et al. Distortion correction of diffusion-weighted magnetic resonance imaging of the head and neck in radiotherapy position. Acta Oncol (2017) 56(11):165963. doi: 10.1080/0284186X.2017.1377347

83. Schakel T, Hoogduin JM, Terhaard CHJ, Philippens MEP. Technical Note: Diffusion-weighted MRI with minimal distortion in head-and-neck radiotherapy using a turbo spin echo acquisition method. Med Phys (2017) 44(8):4188-93. doi: 10.1002/mp.12363

84. Finazzi T, van Sörnsen de Koste JR, Palacios MA, Spoelstra FOB, Slotman BJ, Haasbeek CJA, et al. Delivery of magnetic resonance-guided single-fraction stereotactic lung radiotherapy. Phys Imaging Radiat Oncol (2020) 14:17-23. doi: $10.1016 /$ j.phro.2020.05.002

Conflict of Interest: DT declares institutional collaborations including financial and non-financial support with Elekta, Philips, Siemens, PTW Freiburg, and IBA Dosimetry. DL declares institutional financial support by Varian.

Copyright (C) 2021 Thorwarth and Low. This is an open-access article distributed under the terms of the Creative Commons Attribution License (CC BY). The use, distribution or reproduction in other forums is permitted, provided the original author(s) and the copyright owner(s) are credited and that the original publication in this journal is cited, in accordance with accepted academic practice. No use, distribution or reproduction is permitted which does not comply with these terms. 